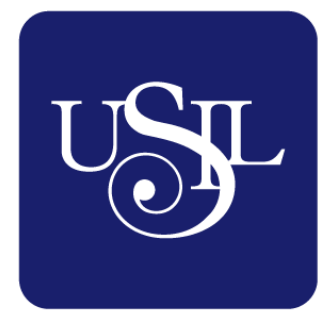

UNIVERSIDAD

SAN IGNACIO

DE LOYOLA

ESCUELA DE POSTGRADO

Maestría en Ciencias Empresariales

\title{
PLAN ESTRATÉGICO DE LA UNIDAD DE NEGOCIOS OPM (OUT PATIENT MARKET) DEL LABORATORIO B. BRAUN MEDICAL PERÚ
}

Tesis para optar el grado de Maestro en Ciencias Empresariales

\section{MARIA CECILIA UGÁS ECHECOPAR LOURDES DOMINGA ZÚÑIGA CONCHA}

\author{
Asesor: \\ Dr. Edmundo Rafael Casavilca Maldonado
}

Lima - Perú

2018 
Índice

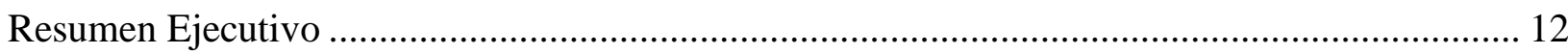

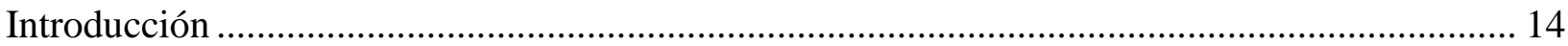

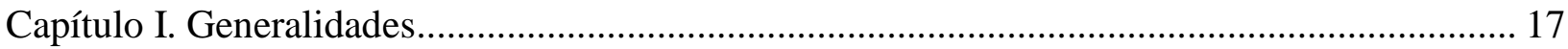

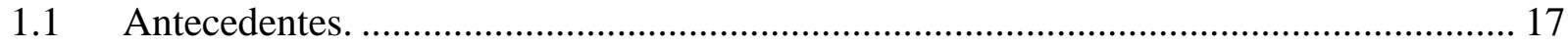

1.2 Determinación del problema u oportunidad........................................................... 19

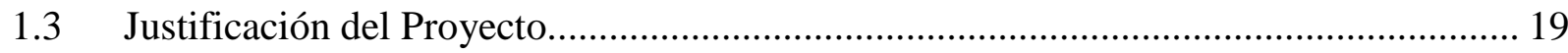

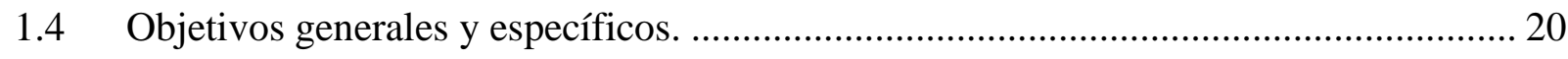

1.5 Alcances y limitaciones de la investigación............................................................ 20

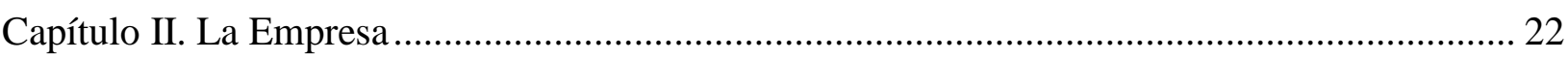

2.1. Antecedentes de la empresa. ..................................................................................... 22

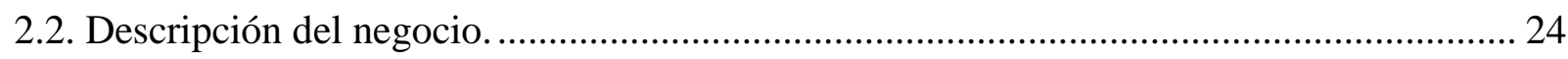

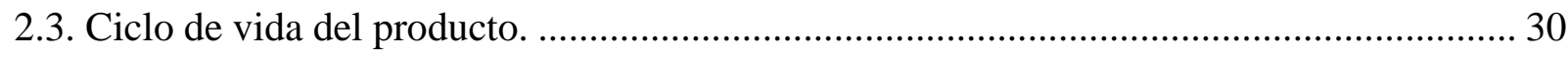

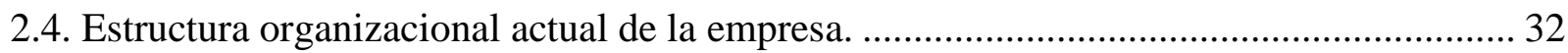

2.5. Situación de Mercado y Financiera actual de la Industria. .............................................. 36

Capítulo III. Formulación de Visión, Misión y Valores de la Empresa ........................................ 40

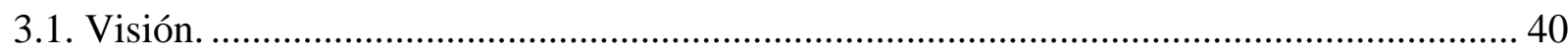

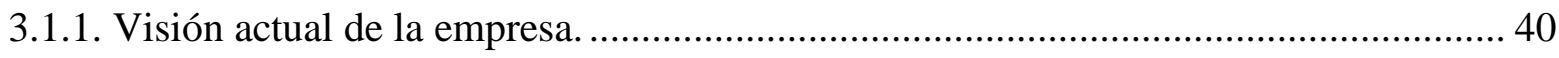

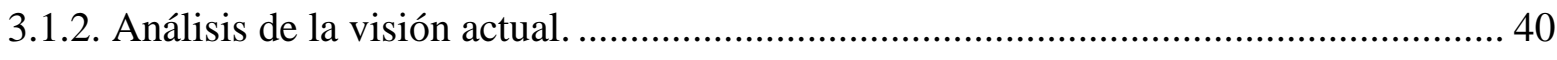

3.1.3. Matriz de la visión propuesta para la empresa. .................................................... 41

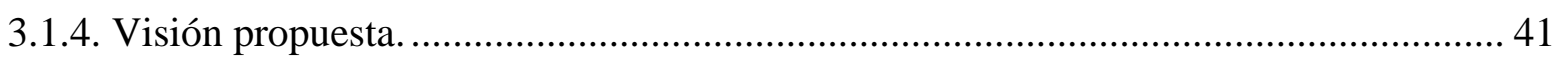

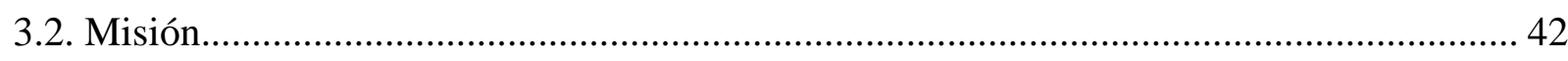

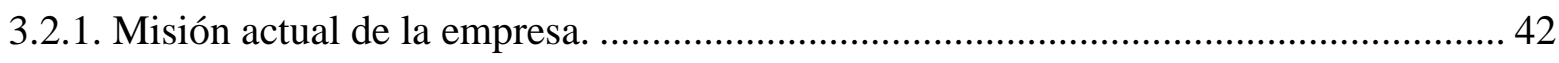

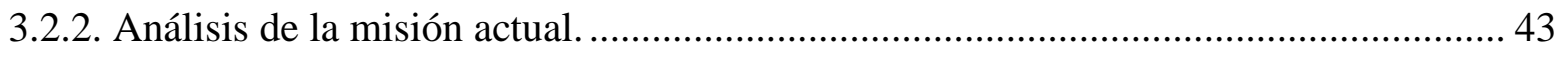

3.2.3. Elementos de la misión propuesta para la empresa................................................ 43

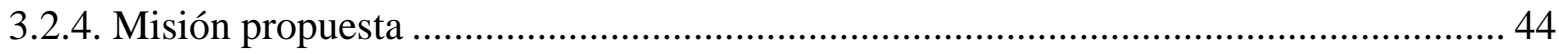

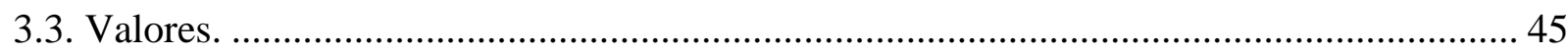




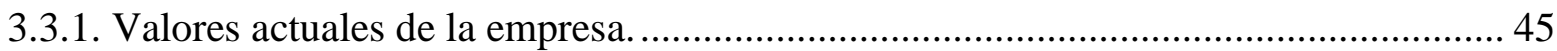

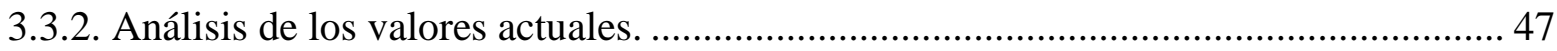

3.3.3. Elementos de los valores propuestos para la empresa. ......................................... 48

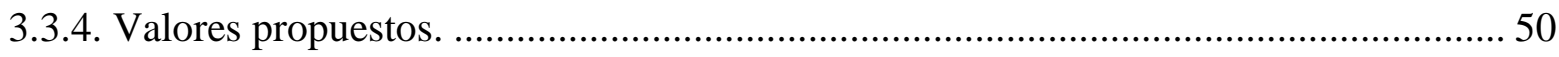

3.4. Alineamiento estratégico de la Visión, Misión y Valores de la empresa........................... 51

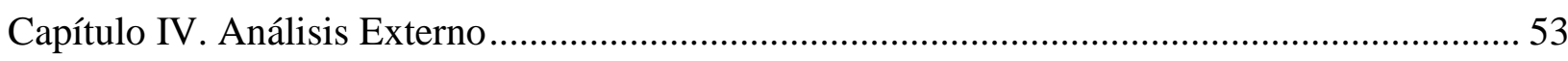

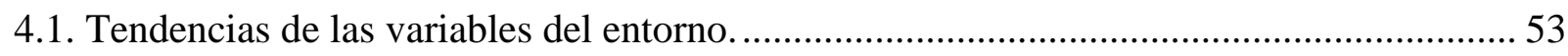

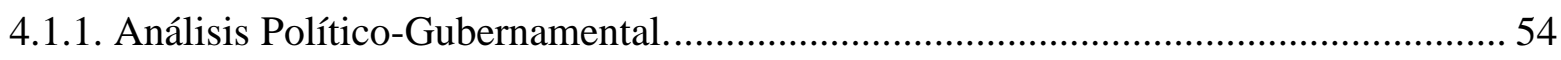

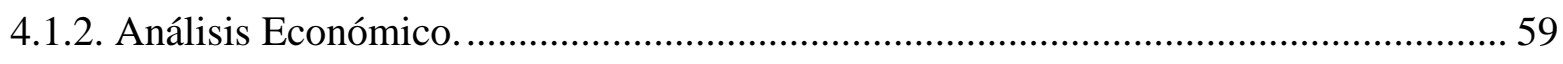

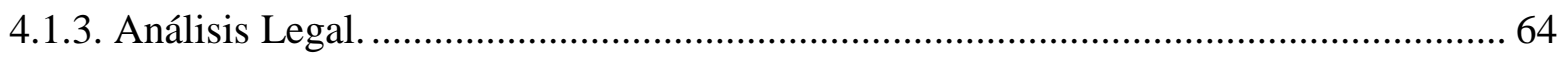

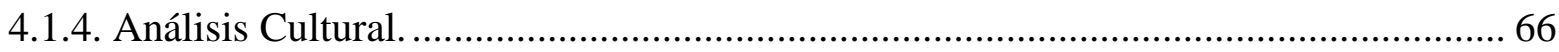

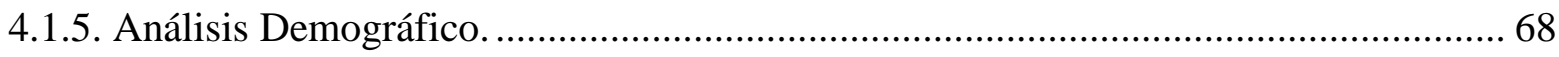

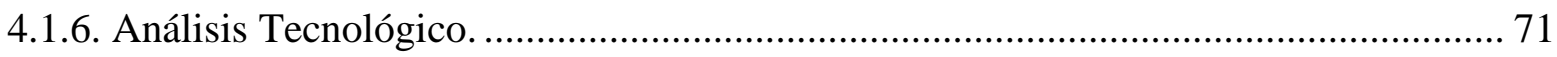

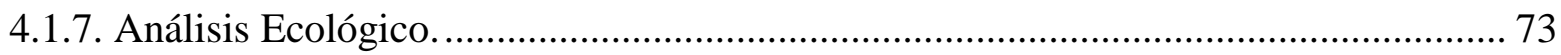

4.2. Impacto en clientes / proveedores de cada una de las variables del entorno. .................... 73

4.3. Efecto en la empresa de cada una de las variables del entorno....................................... 75

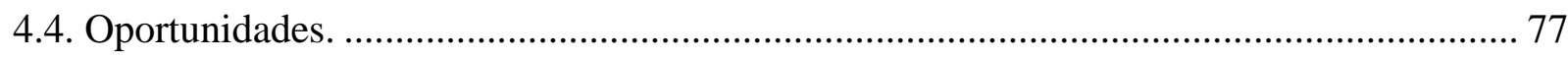

4.5. Matriz de Evaluación de los Factores Externos (EFE). ................................................ 79

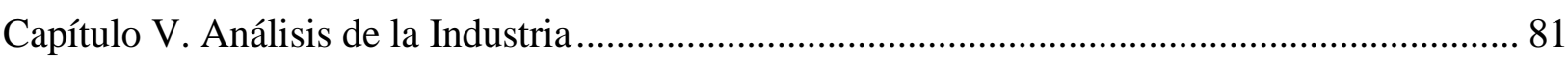

5.1. Descripción del Mercado (demanda) e Industria (oferta). ............................................ 81

5.2. Descripción las cinco fuerzas competitivas de la industria.......................................... 83

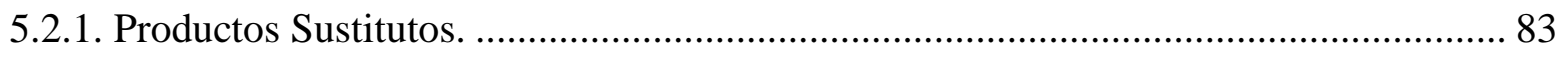

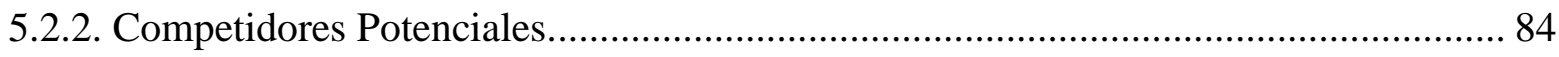

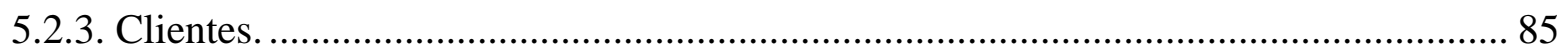

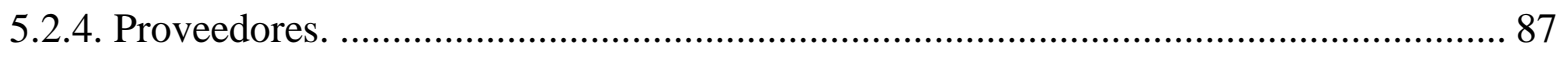

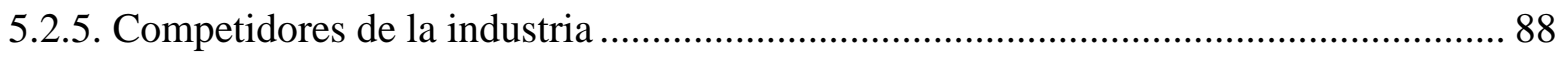

5.3. Matriz de atractividad de cada una de las cinco fuerzas. ............................................... 90 


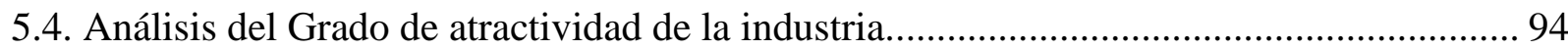

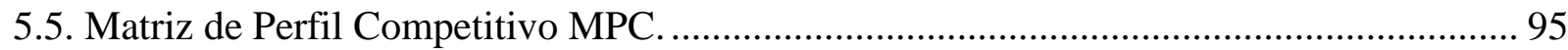

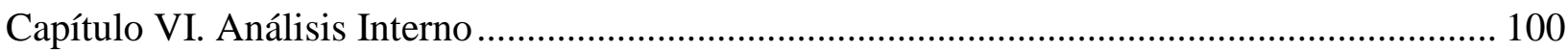

6.1. Descripción de las actividades de la cadena de valor de la empresa............................... 101

6.2. Indicadores de cada una de las actividades de la cadena de valor. ................................ 110

6.3. Benchmarking y comparación con los líderes de la industria de cada una de las

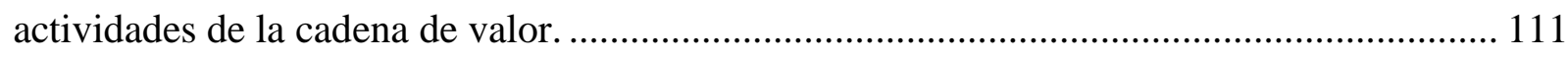

6.4. Determinar las competencias de la empresa. .......................................................... 112

6.5. Identificación y determinación de las ventajas competitivas de la empresa.................... 114

6.6. Matriz de Evaluación de los Factores Internos EFI. ..................................................... 115

Capítulo VII. Formulación de los Objetivos y Diseño de las Estrategias .................................. 117

7.1. Alcance y planteamiento de los objetivos estratégicos. ............................................. 117

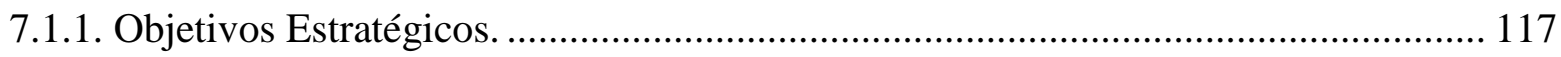

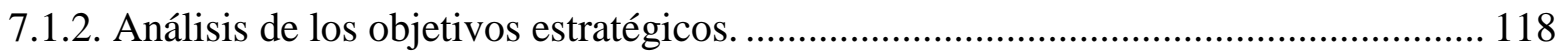

7.2. Diseño y formulación de estrategias. ........................................................................ 120

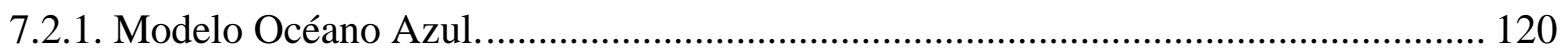

7.2.1.1. Lienzo de la estrategia actual de la empresa................................................. 121

7.2.1.2. Lienzo de la estrategia de la industria........................................................... 122

7.2.1.3. Matriz (eliminar, reducir, incrementar, crear). .......................................... 123

7.2.1.4. Lienzo de la nueva estrategia considerada................................................... 124

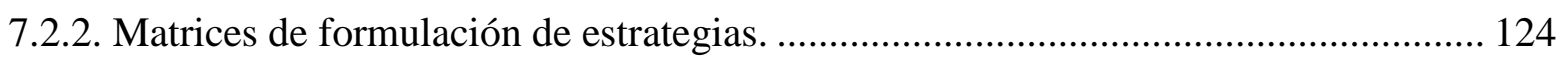

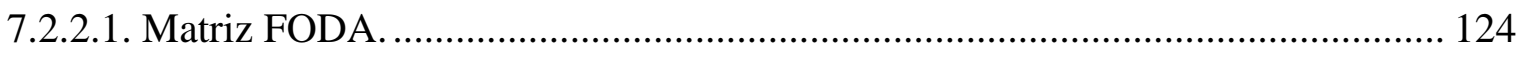

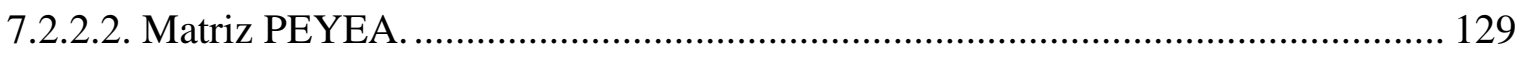

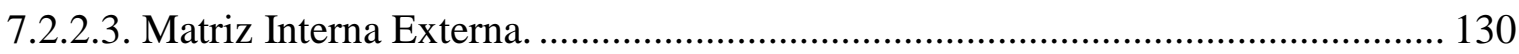

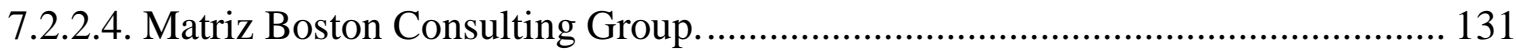

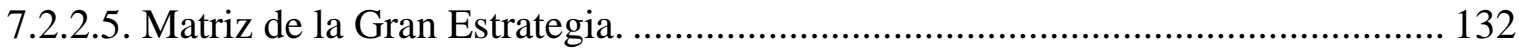

7.3. Resumen de las Estrategias Formuladas. ................................................................. 133 


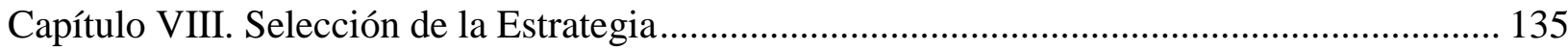

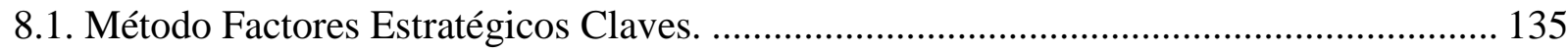

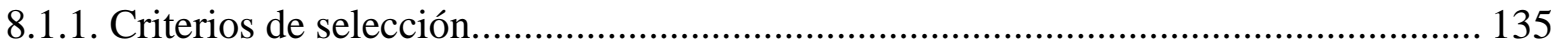

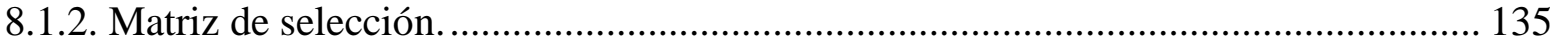

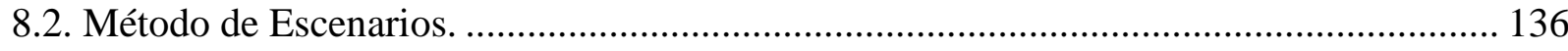

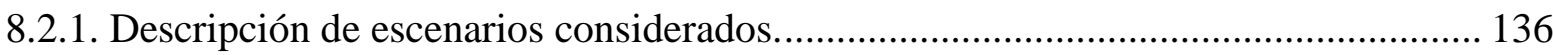

8.2.2. Comparación de Estrategias con escenarios........................................................ 137

8.3. Matriz de Planeación Estratégica Cuantitativa MPEC.................................................. 137

8.4. Descripción de estrategia seleccionada...................................................................... 139

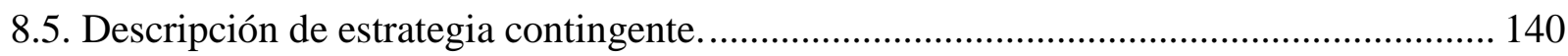

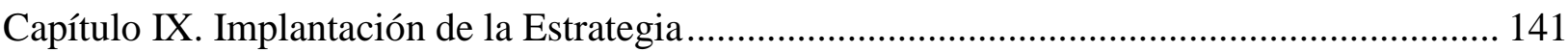

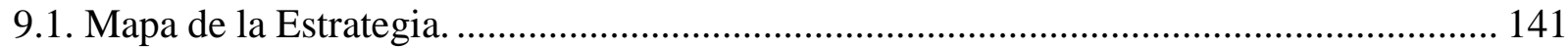

9.2. Objetivos específicos según el mapa de la estrategia................................................. 143

9.3. Indicadores para cada uno de los objetivos específicos. .............................................. 144

9.4. Metas para cada uno de los objetivos específicos...................................................... 145

9.5. Iniciativas (acciones a llevar a cabo para cada uno de los objetivos específicos)

Estrategias, programas, políticas, reglas, procedimiento.................................................... 147

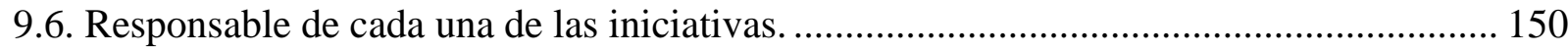

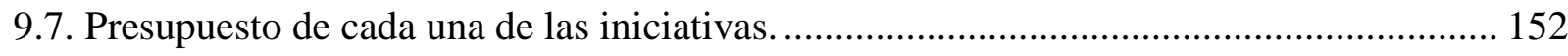

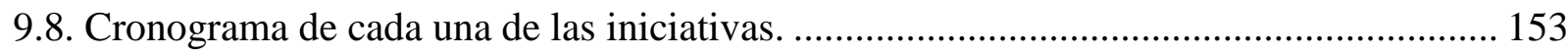

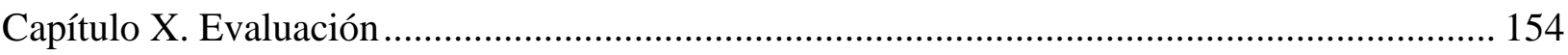

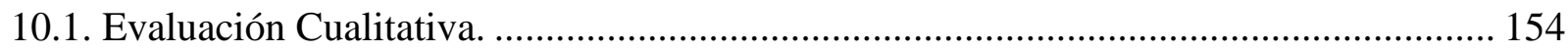

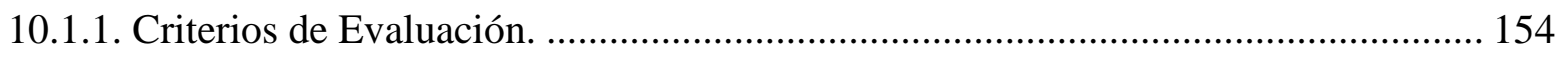

10.1.2. Comparación de la estrategia con los criterios. ............................................... 155

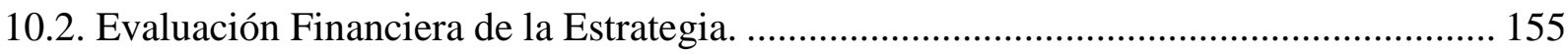

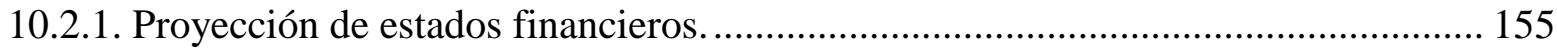

10.2.2. Estado de resultados (situación actual y con la nueva estrategia)......................... 159 
10.2.3. Balance general (situación actual y con la nueva estrategia). ................................ 160

10.2.4. Flujo de efectivo (situación actual y con la nueva estrategia)............................... 163

10.2.5. Evaluación Financiera (VAN, TIR y ratios financieros)....................................... 164

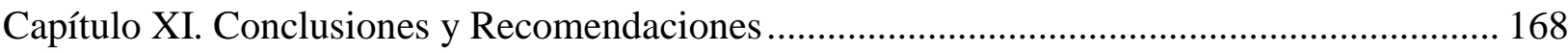

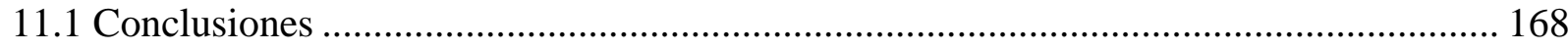

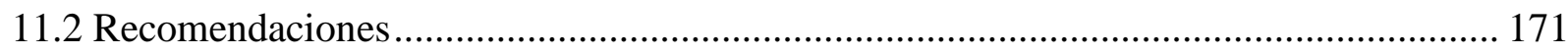

ANEXO 01: Entrevista en Profundidad a CEO ............................................................... 172

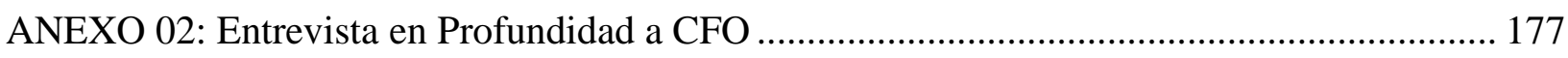

ANEXO 03: Entrevista en Profundidad a Profesional Experto ................................................ 182

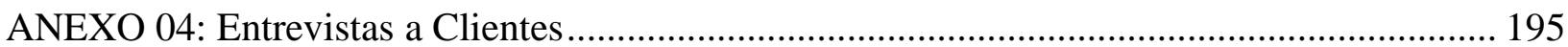

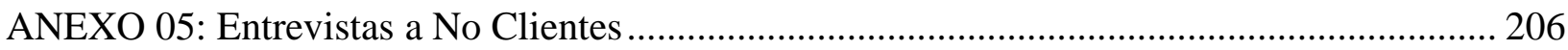

ANEXO 06: Panel de Discusión sobre Clima Laboral ........................................................... 213

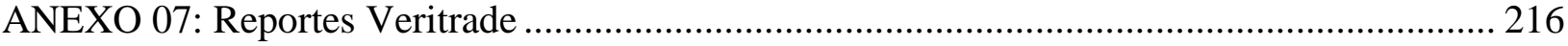

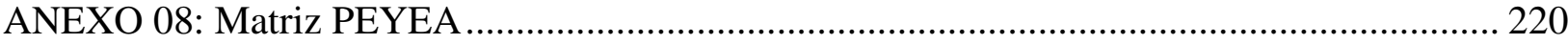

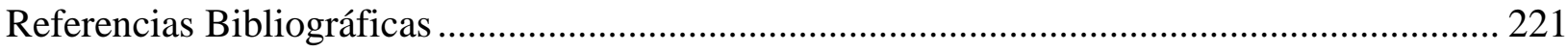




\section{Índice de Figuras}

Figura 1. B. Braun History. ...................................................... 22

Figura 2. B. Braun en el mundo.................................................. 23

Figura 3. B. Braun Medical Perú, Lurín Lima.......................................... 24

Figura 4. Productos de la línea cuidados de la piel............................... 27

Figura 5. Productos de la línea ostomía.......................................... 28

Figura 6. Flujo grama de procesos internos OPM................................. 30

Figura 7. Ventas y Utilidad OPM............................................. 32

Figura 8. Organigrama B. Braun Medical Perú........................................ 34

Figura 9. Organigrama Unidad de Negocios OPM............................... 34

Figura 10. Fabricantes de Dispositivos Médicos................................ 37

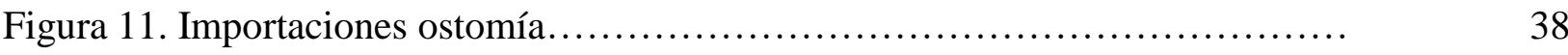

Figura 12. Importaciones cuidado de heridas. ................................... 39

Figura 13. Importaciones División OPM.......................................... 39

Figura 14. Valores de B. Braun Medical Perú......................................... 47

Figura 15. Modelo Triaxial de la Dirección por Valores. ............................ 49

Figura 16. Arquitectura estratégica para la gestión del conocimiento basada en la dirección por valores................................................... 50

Figura 17. Alineación de Valores, Visión y Misión. ................................ 52

Figura 18. Foto durante la elaboración y el análisis de la Misión, Visión y Valores......... 52

Figura 19. Diseño del Medio ambiente Externo. ................................. 54

Figura 20. Población con cobertura de salud.................................... 55

Figura 21 Sistema de salud................................................... 56

Figura 22 Crecimiento anual del PBI........................................... 59

Figura 23. Gráfico comparativo del Gasto en Salud como Porcentaje del PBI en los principales países de América del Sur.......................................... 60

Figura 24.Estructura del Gasto Real Per Cápita, según grupo de gasto, 2009-2015....... 61 
Figura 25. Variación Porcentual del Gasto Real Per Cápita, según grupo de gasto $2015 / 2014$

Figura 26. Evolución del tipo de cambio de Junio 2012 a Mayo 2016

Figura 27. Pasos para obtener un registro sanitario de dispositivo médico.

Figura 28. Importaciones de Dispositivos médicos

Figura 29. Diagrama de las 5 fuerzas de Porter...

Figura 30. Segmentación de los clientes en Perú....

Figura 31. Competidores en el mercado de Stoma Care

Figura 32. Competidores en el mercado de Wound Management........................ 90

Figura 33. Participación de Mercado Grupo Estratégico A. Fuente Veritrade............... 96

Figura 34. Grupos Estratégicos................................................... 97

Figura 35. Cadena de Valor......................................................... 100

Figura 36. Proceso de Logística interna......................................... 101

Figura 37. Proceso de Logística externa........................................... 103

Figura 38. Proceso Adquisiciones............................................... 106

Figura 39. Cuadro comparativo con los líderes de la industria........................ 112

Figura 40. Lienzo de Estrategia Actual.......................................... 122

Figura 41. Lienzo de Estrategia de la Industria.................................... 123

Figura 42. Matriz Eliminar, Reducir, Incrementar, Crear............................ 123

Figura 43. Lienzo de Nueva Estrategia......................................... 124

Figura 44. Matriz PEYEA....................................................... 130

Figura 45. Matriz IE ............................................................ 131

Figura 46. Matriz BCG......................................................... 132

Figura 47. Matriz GE............................................................... 133

Figura 48. Mapa Estratégico...................................................... 142

Figura 49. Cronograma de implementación de actividades............................ 153 


\section{Índice de Tablas}

Tabla 1 Resumen de las características, objetivos y estrategias del ciclo de vida de los productos

Tabla 2 Clientes, Ventas y Utilidades de la Unidad OPM.

Tabla 3 Importaciones Anuales en Dólares por Línea de Producto.

Tabla 4 Matriz de la Visión.

Tabla 5 Elementos de la misión propuesta para la Unidad de Negocios OPM

Tabla 6 Gasto en Salud como Porcentaje del PBI y Per Cápita

Tabla 7 Encuesta de Expectativas Macroeconómicas: Tipo de Cambio (PEN por USD)....

Tabla 8 Proyección de la Población en Perú

Tabla 9 Población en Perú por Provincia.

Tabla 10 Impacto de las Oportunidades y Amenazas del Macro Entorno en Cliente y

Proveedores

Tabla 11 Efecto en la Empresa de las Variables del Macro Entorno. ...

Tabla 12 Oportunidades y Amenazas de las Variables del Macro Entorno.

Tabla 13 Matriz EFE.

Tabla 14 Proyección del Mercado de Dispositivos Médicos peruano. 2014-2019.

Tabla 15 Clientes por Sub Sector.

Tabla 16 Competidores Ostomía.

Tabla 17 Competidores Cuidado de Heridas

Tabla 18 Matriz de Atractividad: Amenaza de Productos Sustitutos.

Tabla 19 Matriz de Atractividad: Amenaza de Nuevos Competidores

Tabla 20 Matriz de Atractividad: Poder de Negociación de Proveedores.

Tabla 21 Matriz de Atractividad: Poder de Negociación de Clientes. ... 
Tabla 23 Matriz de Atractividad de la Industria...

Tabla 24 Competidores según grupos estratégicos

Tabla 25 Matriz de Perfil Competitivo (MPC).

Tabla 26 Actividades apoyadas por Infraestructura.

Tabla 27 Indicadores de Actividades Primarias

Tabla 28 Indicadores de Actividades de apoyo

Tabla 29 Matriz VRIO.

Tabla 30 Matriz EFI.

Tabla 31 La Estrategia del Océano Rojo Frente a la Estrategia del Océano Azul.

Tabla 32 Estrategia Océano Rojo Vs. Océano Azul

Tabla 33 Matriz FODA

Tabla 34 Input para matiz BCG.

Tabla 35 Matriz de Decisión Estratégica.

Tabla 36 Comparación de Estrategias y Escenarios.

Tabla 37 Matriz Cuantitativa de Planeamiento Estratégico

Tabla 38 Detalle de Indicadores por objetivo

Tabla 39 Detalle de Metas por Indicador

Tabla 40 Responsables de Iniciativas.

Tabla 41 Presupuesto de Iniciativas expresado en PEN.

Tabla 42 Matriz de Rumelt

Tabla 43 Estado de Resultados OPM proyectado con la situación actual.

Tabla 44 Estado de Resultados OPM proyectado con la nueva estrategia

Tabla 45 Balance General OPM proyectado con la situación actual.

Tabla 46 Balance General OPM proyectado con la nueva estrategia.

Tabla 47 Flujo de Efectivo OPM proyectado con la situación actual. 
Tabla 48 Flujo de Efectivo OPM proyectado con la nueva estrategia..................... 163

Tabla 49 Costo de Capital....................................................... 164

Tabla 50 Evaluación Económica.................................................. 166

Tabla 51 Ratios Financieros con la situación actual................................ 167

Tabla 52 Ratios Financieros con la nueva estrategia................................ 167 